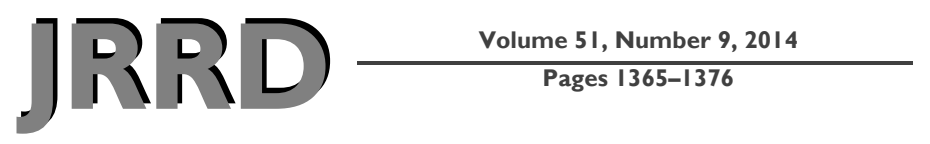

\title{
Effect of interpolation on parameters extracted from seating interface pressure arrays
}

\author{
Michael Wininger, PhD; ${ }^{1-2}$ Barbara Crane, PhD, PT $^{\mathbf{2}^{*}}$ \\ ${ }^{1}$ Cooperative Studies Program, Department of Veterans Affairs Connecticut Healthcare System, West Haven, CT; \\ ${ }^{2}$ Department of Rehabilitation Sciences, University of Hartford, West Hartford, CT
}

\begin{abstract}
Interpolation is a common data processing step in the study of interface pressure data collected at the wheelchair seating interface. However, there has been no focused study on the effect of interpolation on features extracted from these pressure maps, nor on whether these parameters are sensitive to the manner in which the interpolation is implemented. Here, two different interpolation paradigms, bilinear versus bicubic spline, are tested for their influence on parameters extracted from pressure array data and compared against a conventional low-pass filtering operation. Additionally, analysis of the effect of tandem filtering and interpolation, as well as the interpolation degree (interpolating to 2, 4, and 8 times sampling density), was undertaken. The following recommendations are made regarding approaches that minimized distortion of features extracted from the pressure maps: (1) filter prior to interpolate (strong effect); (2) use cubic interpolation versus linear (slight effect); and (3) ensure nominal difference between interpolation orders of 2, 4, and 8 times (negligible effect). We invite other investigators to perform similar benchmark analyses on their own data in the interest of establishing a community consensus of best practices in pressure array data processing.
\end{abstract}

Key words: data processing, filtering, force, interpolation, pressure array, pressure map, pressure ulcer, seating, sitting, wheelchair.

\section{INTRODUCTION}

The force-sensing array has a long-recognized utility for not only scientific inquiry [1], but also clinical care.
Pressure mapping has come to be regarded as a "best practice" in prevention of pressure ulcers in the clinical care setting [2]. While the pressure map is a useful tool for visualization and qualitative assessment of the pressure distribution across the seating surface, extraction of quantitative data from pressure arrays is becoming increasingly common. While there remain many significant reservations in the scientific community about the use of pressure mapping as a quantitative modality, it is becoming increasingly popular in the study of the seating interface. For example, expression of the interface pressure via the dispersion index allows for objective measure of the evenness of load distribution and has been used to inform clinical recommendations [3]. Features extracted from pressure maps have been useful in a wide variety of clinical applications relevant to wheelchair use, particularly in pressure ulcer prevention and management [4-6]. Indeed, objective measures derived from pressure array data are lately considered sufficiently robust as to be utilized as primary outcome measures in randomized clinical trials [7-8]. A great need exists to continue to develop reliable and sensitive parameters and to refine methods for processing and analyzing these data.

\footnotetext{
Abbreviations: FSA = force sensitive application, RMS = root-mean-square.

*Address all correspondence to Barbara Crane, PhD, PT; University of Hartford, West Hartford, CT 06117; 860-7685371; fax: 860-768-4558. Email: bcrane@hartford.edu http://dx.doi.org/10.1682/JRRD.2014.04.0101
} 
Whereas metrics extracted from pressure array data are sensitive to the methods by which the arrays are processed, special care must be taken to understand the outcome of each processing step and how it might alter the measurement. Furthermore, while the approaches for processing pressure array data vary widely, it is possible that the amount of information that can be integrated across studies would be greatly increased by a robust understanding of the effect of each link in the signal processing chain [9]. Here, focused study is applied to one particular processing step that is endemic to the analysis of pressure array data, i.e., interpolation, in the interest of better understanding the effect of the degree and ordering of an interpolation transformation on conventional features extracted from pressure maps.

Interpolation is a technique used to estimate data points where no data were collected [10]. In particular application to pressure array data used for assessment of the interface pressure distribution while seated, interpolation has the effect of increasing sampling resolution of a low spatial resolution measurement modality. A great many of the commercially available pressure mats contain only 256 sensors in a $16 \times 16$ grid, and interpolation provides a smoother, more interpretable high-resolution image of the buttock anatomy. Interpolation is a method de rigueur in the study of seat pressure and has been widely used for the purposes of both visualization and prediction for more than $25 \mathrm{yr}$ [11].

There are many different interpolation techniques, and a comprehensive review is beyond the scope of this work. However, two approaches have emerged as most commonly used in the analysis of seating data: linear and cubic spline. In short summary, the linear (more formally, bilinear interpolation in the context of two-dimensional array data) involves a simple point-slope imputation of data at points intermediate to true observation; the cubic spline (more formally, bicubic spline) involves a summed, iterative approximation through fitting a thirdorder polynomial [12]. Naturally, the method by which a data set is interpolated will affect the values of the interpolants. Different methods of interpolation may also alter the values of the observed data (acting, in a way, as a separate filter).

For example, in a single-dimensional data set extracted from a row of sensors in a $16 \times 16$ pressure mat, 16 sensors are interpolated out to 31 sensors via linear (Figure (a)) and cubic (Figure (b)) interpolation. It is evident from the Figure that three simple descriptive sta- tistics extracted from the interpolated data differ somewhat from the features extracted from the raw data. Feature extraction from pressure maps is a common practice, e.g., pursuant to some estimation of pressure ulcer risk [13-16]. Indeed, these variables have thorough representation in the literature, including studies invoking both the linear [17-19] and cubic [20-22] interpolation. Furthermore, data can be interpolated to any degree: once (e.g., 16-31 data points per row), twice (e.g., to 61 data points), or three times (e.g., $121 \times 121$ data points) are all typical. In addition to the effects of interpolation, any filtering operation will further alter the data, and the effect of tandem interpolation plus filtering might be different depending on the order of operations. Whereas equally abundant examples exist of both interpolation preceding a filtering [23-25] and interpolation following a filtering [26-28], the order of operations appears to be worthy of further study.

We set out to measure the effect of linear versus cubic spline interpolation on these simplistic pressure map features, pursuant to a better understanding of the sensitivity of common outcome measures to the antecedent signal processing methodologies. This was accomplished through comparison of the maximum and average pressure and root-mean-square (RMS) of the pressure gradient of both the raw, unprocessed array data and data interpolated with a bilinear versus bicubic spline. The primary null hypotheses were (1) bilinear interpolation distorts these features to an extent that yields statistical significance, (2) bicubic interpolation distorts these features to an extent that yields statistical significance, (3) the distortion of these features due to bicubic interpolation is not significantly different from that due to bilinear interpolation, and (4) the distortion of these features due to either interpolation approach (linear or cubic) is not significantly different from that due to conventional lowpass filtering. Secondary objectives were to study the effects on these parameters of order of operations (filterthen-interpolate vs interpolate-then-filter) and interpolation degree (interpolation of a $16 \times 16$ sensor array to a $31 \times 31,61 \times 61$, and $121 \times 121$ array). The outcomes of these hypothesis tests will inform interpretation of as yet collected data and will facilitate future study design. 
(a)

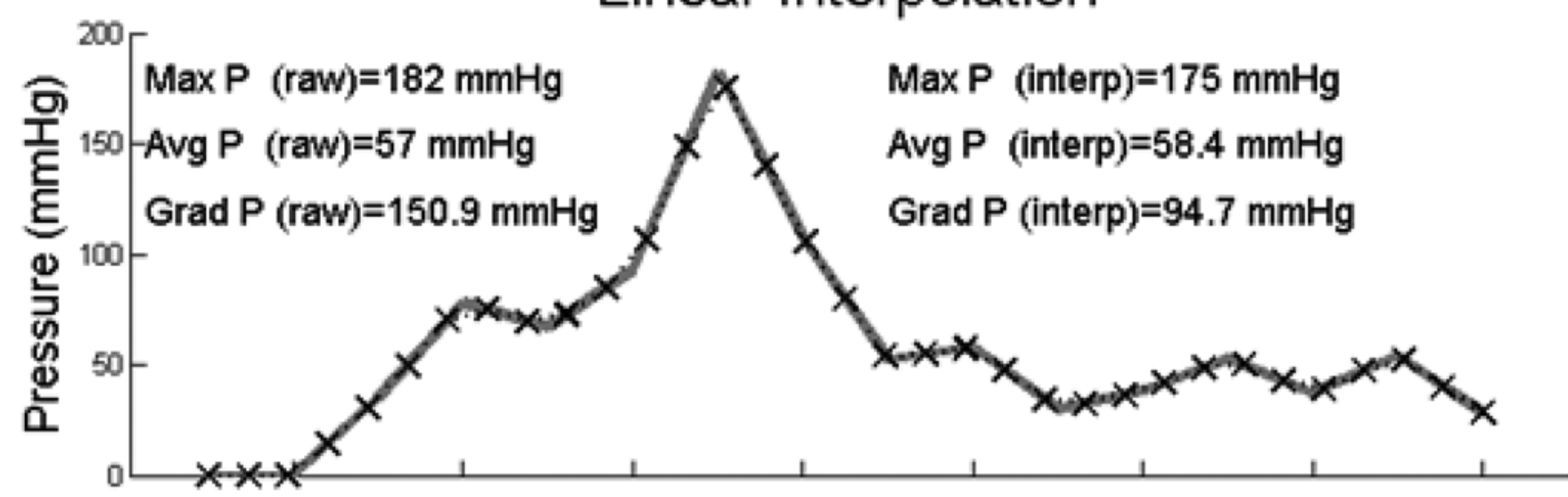

(b)

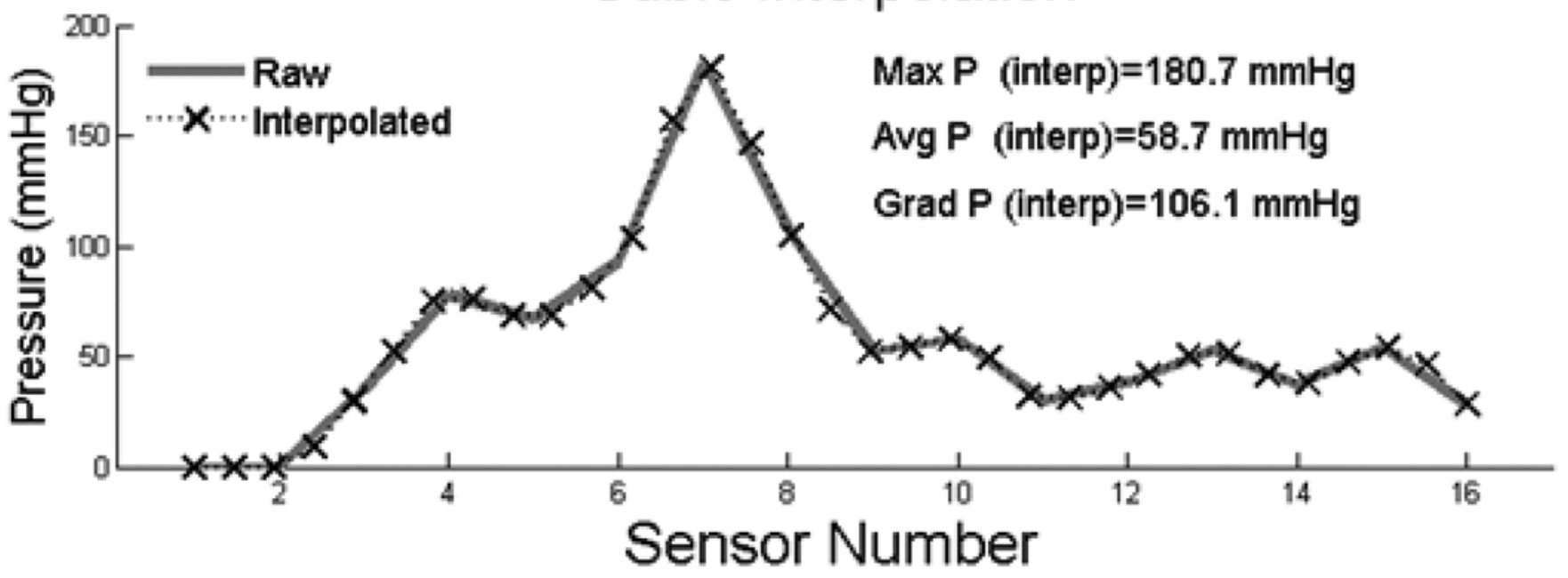

Figure.

Effect of two different interpolation techniques ((a) linear and (b) cubic) on row of sensors in seating interface pressure array. Peak value (corresponding with pressure beneath ischial tuberosity), average (Avg) pressure, and root-mean-square of pressure gradient (Grad) within row are shown for both raw and interpolated (interp) data. Max = maximum.

\section{METHODS}

\section{Participants}

In this study, 22 volunteers were recruited from six long-term care centers, of whom 21 were current wheelchair users and 1 recently transitioned to supervised ambulation. The study entrance criteria were purposefully minimal: there were no restrictions on age or health status, and there was no restriction placed on the style of wheelchair, cushion, or positioning supports (towels, blankets, wedges, etc.) preferred by the participants.
Whereas this study was essentially methodological in nature, and no hypotheses were devised with regard to interventional therapies, we sought a maximally generalizable population with broad clinical relevance. However, in the interest of safety, participants were screened for current pressure ulcer at the time of data collection. Table 1 shows study demographics.

We note that participants were required to tolerate sitting for the entire duration of the observation period (generally $<20 \mathrm{~min}$ ). All participants were oriented to the study and provided informed consent prior to data collection. 
Table 1.

Participant demographics.

\begin{tabular}{lc}
\hline \multicolumn{1}{c}{ Descriptor } & n or Mean \pm SD \\
\hline Age (yr) & $80 \pm 10$ \\
Height (in.) & $65 \pm 5$ \\
Weight (lb) & $165 \pm 44$ \\
Sex & 11 \\
$\quad$ Female & 11 \\
$\quad$ Male & \\
Wheelchair Type & 15 \\
$\quad$ Standard Manual or Hemi-Height & \\
$\quad$ Manual & 7 \\
$\quad$ Custom Manual Tilt or Power & \\
Cushion Type & 8 \\
Foam & 6 \\
Air-Filled & 4 \\
Foam + Gel Combination & 4 \\
Other & \\
\hline \hline
\end{tabular}

Methods for this study were approved by the University of Hartford Human Subjects Committee and the Genesis Health Care System Institutional Review Board.

\section{Data Collection}

Seat interface pressure data were collected via the force sensitive application (FSA) system (Vista Medical Ltd; Winnipeg, Canada). This mat array contained a $16 \times$ 16 sensor grid (sensel size of $2.38 \times 2.38 \mathrm{~cm}$ with $3.1 \mathrm{~mm}$ spacing between sensors). The pressure mat was placed in an isolation bag to allow for cleaning with a disinfecting agent between sessions. Prior to data collection, the FSA mat was calibrated per protocol to the standard $200 \mathrm{~mm} \mathrm{Hg}$ maximum, according to the manufacturer's instructions. The pressure mat was placed as close to the participant as possible, i.e., no blankets or other support materials were placed between the mat and the buttocks; participants wore their normal clothing; and incontinence protection garments were allowed and not controlled for in this study.

Once positioned in the wheelchair, participants were instructed to sit as still as possible for 6 min to allow for appropriate settling time and to allow for sensor creep $[4,29]$. Interface pressure data were recorded at $1 \mathrm{~Hz}$ for 2 min, yielding a total of approximately 120 samples per participant.

\section{Data Processing}

This study's primary objective related to two signal processing operations: interpolation and filtering. All processing was performed on raw data obtained from the FSA software and within the MATLAB (R2012a, MathWorks; Natick, Massachusetts) numerical computing environment. A single data conditioning step was implemented prior to processing: sensors with less than $5 \mathrm{~mm} \mathrm{Hg}$ were considered noise and were overwritten as zero. The interpolation operations were either bilinear or bicubic spline interpolation, to an order of $1 \times, 2 \times$, or $3 \times$, and were performed via the "interp2" command. The filtering was of a low-pass type via standard Gaussian neighborhood operator, of size commensurate with 20 percent of the array width: a $16 \times 16$ array was filtered with a $3 \times 3$ Gaussian, a $31 \times 31$ array was filtered with a $6 \times 6$ Gaussian, and so on. In this way, all data were filtered homogenously, yielding the most direct comparison possible between different array sizes. Data were processed in order (either filter-then-interpolate or interpolate-thenfilter) per design.

\section{Data Analysis and Hypothesis Testing}

From each processed array of sensor data, three scalar measures were calculated: (1) maximum pressure, (2) average pressure, and (3) RMS pressure gradient. While we sought to incorporate conventional measures that would likely be interpretable (if not familiar) to a broad audience, it was necessary to make two modest modifications that will aid in reproducibility of this work: the average pressure was performed over only those sensors with a nonzero value, and the commonly used gradient measure was converted to a scalar (nonmatrix) value by summing over the point-wise gradient [30].

The effect of the various processing steps on these parameters was assessed via a simple percent-change comparison. In most cases, the difference between the values of parameters extracted from the processed array was subtracted from the values of parameters extracted from the unprocessed (raw) array and normalized to the raw value (and thus expressed as a proportion of the original value). The single exception to this design is in the analysis of the effect of operational ordering (filter-theninterpolate vs interpolate-then-filter), where the processed data were compared directly against the raw data filtered (but not interpolated). This is the more informative comparison in assessing the effect of interpolation as a compound processing step onto data that are intended 
to be filtered a priori, not per se the effect of interpolation + filtering against raw data. Hypothesis tests were then performed either against raw (deviation from unity, tested via Wilcoxon rank-sum) or between conditions (pairwise $t$-test).

\section{RESULTS}

\section{Primary Objective}

The primary objective of this investigation was to determine the effect of linear and cubic interpolation on features extracted from low-resolution pressure arrays versus a conventional low-pass filtering operation. Here it was found that, except for maximum pressure following linear interpolation, both linear and cubic interpolation do indeed significantly alter the features extracted from pressure maps (hypotheses 1 and 2). The effects were mixed: small effect on maximum pressure $(0 \%$ and $1 \%$ increase), modest effect on pressure gradient (5\% and $1 \%$ decrease), and moderate effect on mean pressure ( $9 \%$ and $11 \%$ decrease).

Furthermore, there is a significant difference of the effect of linear versus cubic interpolation in all three parameters. Although the effects are mixed, with less extreme effects of linear interpolation for maximum and mean pressure and more extreme effects of linear interpolation for pressure gradient (hypothesis 3 ), in all cases, the relative effect was small. Last, we report that the features extracted from interpolated arrays were significantly less deviated from the features extracted from the raw data than were those following low-pass filter (hypothesis 4). In all cases, the effect was large (between $17 \%-35 \%$ change in parameter value via filtering vs $0 \%-$ 11\% change via interpolation) (Table 2).

\section{Compound Processing and Order of Operations}

As a secondary objective, we sought to measure the net effect of interpolation and filtering as tandem operations. This was accomplished by measuring the change in the pressure array parameters following (1) filtering and then interpolation and (2) interpolation and then filtering; interpolation was via either linear or cubic approaches and filtering was via Gaussian filter of 20 percent length.

Table 3 shows the results.

We observed that in the case of interpolation after filtering, the deviation from baseline (filtering-withoutinterpolation) is negligible: generally a 0 to 2 percent difference, with the exception of mean pressure in cubic interpolation (8\% difference). In contrast, filtering after interpolation greatly alters the data relative to baseline: differences of 14 to 31 percent, with the exception of mean pressure in linear interpolation (average difference near 0\%) (Table 3).

Furthermore, there is generally little difference between linear and cubic interpolation when the filter is applied first (again with the exception of mean pressure): absolute differences of 1,8 , and 1 percent in the three parameters (column 2 minus column 1 in Table 3). These differences increase substantially when the interpolated data are then filtered (4\%, 14\%, and 7\%; column 4 minus column 3 in Table 3).

Table 2.

Difference in pressure array parameters following interpolation via linear versus cubic spline; change following low-pass filtering shown as baseline comparator.

\begin{tabular}{|c|c|c|c|}
\hline \multirow{2}{*}{$\begin{array}{l}\text { Pressure Array } \\
\text { Parameters }\end{array}$} & \multicolumn{3}{|c|}{ Change in Value from Raw (mean \pm SD) } \\
\hline & Interpolated $1 \times{\text { (Linear })^{a}}^{a}$ & Interpolated $1 \times{\text { (Cubic })^{b c}}^{b c}$ & $\begin{array}{c}\text { Low-Pass Filter } \\
(3 \times 3 \text { Gaussian })\end{array}$ \\
\hline Maximum Pressure & $0.00 \pm 0.00^{*}$ & $0.01 \pm 0.02^{\dagger \dagger}$ & $-0.17 \pm 0.09^{\dagger \dagger}$ \\
\hline Mean Pressure & $-0.09 \pm 0.04^{\dagger}$ & $-0.11 \pm 0.05^{\dagger^{*}}$ & $-0.35 \pm 0.10^{\dagger \dagger}$ \\
\hline Pressure Gradient & $-0.05 \pm 0.01^{\dagger}$ & $-0.01 \pm 0.01^{\dagger \dagger}$ & $-0.30 \pm 0.06^{\dagger \dagger}$ \\
\hline 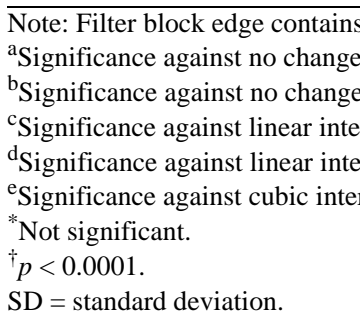 & $\begin{array}{l}\text { of elements as pressure array edge } \\
\text { ter (hypothesis 1). } \\
\text { ter (hypothesis 2). } \\
\text { yypothesis 3). } \\
\text { nypothesis 4). } \\
\text { ypothesis 4). }\end{array}$ & $3 \times 3$ window in $16 \times 16$ array) & \\
\hline
\end{tabular}


Table 3.

Difference in pressure array parameters following linear versus cubic spline interpolation (1×), with low-pass filtering preceding or following interpolation step, as compared with raw data filtered but without interpolating.

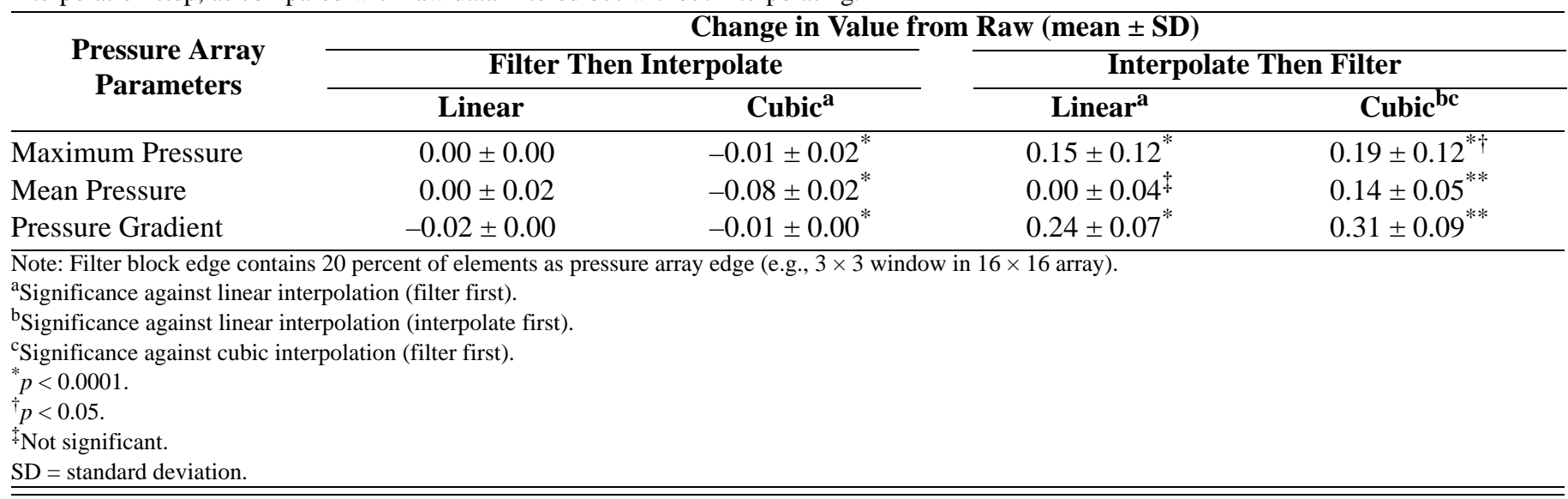

\section{Interpolation Degree}

A third analysis was performed, wherein the degree of interpolation was increased from single interpolation (i.e., interpolation of a $16 \times 16$ array to a $31 \times 31$ array) to a double $(63 \times 63$ array) and triple $(127 \times 127$ array $)$ interpolation. A modest increase in the effect on the extracted features with each increase in interpolation degree was found, e.g., difference in mean pressure of $-0.09 \pm 0.04$ (linear $1 \times$, Table 2), $-0.13 \pm 0.06$ (linear $2 \times$, Table 4), and $-0.15 \pm 0.06$ (linear $3 \times$, Table 4).

This effect is incremental and of minimal effect on feature extraction: the absolute change in parameter value was between 0 and 6 percent following the increase in the degree of interpolation.

\section{DISCUSSION}

\section{Primary Findings}

In interpretation of the effect of these processing operations, we refer the reader to the benchmark of measurement error of the sensor in static loading conditions. In loading conditions similar to those used in this study (ranging up to approximately $200 \mathrm{~mm} \mathrm{Hg}$ ), measurement error typically ranges from 5 to 10 percent [31-33]. In this

Table 4.

Difference in pressure array parameters following linear versus cubic spline interpolation of higher order; data were not filtered.

\begin{tabular}{|c|c|c|c|c|}
\hline \multirow{2}{*}{$\begin{array}{c}\text { Pressure Array } \\
\text { Parameters }\end{array}$} & \multicolumn{4}{|c|}{ Change in Value from Raw (mean \pm SD) } \\
\hline & Linear $(2 \times)^{a}$ & Linear $(3 \times)^{b}$ & Cubic $(2 \times)^{b c}$ & Cubic $(3 \times)^{\text {de }}$ \\
\hline Maximum Pressure & $0.00 \pm 0.00^{*}$ & $0.00 \pm 0.00^{*}$ & $0.02 \pm 0.02^{\dagger \ddagger}$ & $0.02 \pm 0.02^{\ddagger \dagger}$ \\
\hline Mean Pressure & $-0.13 \pm 0.06^{\mathbb{\pi}}$ & $-0.15 \pm 0.06^{\dagger}$ & $-0.15 \pm 0.06^{\S \dagger}$ & $-0.16 \pm 0.07^{\dagger \dagger}$ \\
\hline Pressure Gradient & $-0.07 \pm 0.02^{\ddagger}$ & $-0.07 \pm 0.02^{\dagger}$ & $0.01 \pm 0.01^{\text {丮 }}$ & $0.01 \pm 0.01^{\text {\#† }}$ \\
\hline
\end{tabular}

Note: Filter block edge contains 20 percent of elements as pressure array edge (e.g., $3 \times 3$ window in $16 \times 16$ array).

${ }^{a}$ Significance against linear interpolation (1×).

${ }^{b}$ Significance against linear interpolation $(2 \times)$.

${ }^{\mathrm{C}}$ Significance against cubic interpolation (1×).

${ }^{\mathrm{d}}$ Significance against linear interpolation $(3 \times)$.

${ }^{\mathrm{e} S i g n i f i c a n c e}$ against cubic interpolation $(2 \times)$.

* Incalculable.

${ }^{\dagger}$ Not significant.

${ }^{\ddagger} p<0.0001$.

$\uparrow_{p}<0.001$.

$\S_{p}<0.01$.

$\mathrm{SD}=$ standard deviation. 
context, and following the results presented in Table 2, we concluded that interpolation generally affects the features extracted from pressure maps, but the effects are not extreme. Only in mean pressure do the changes in value approach 10 percent (hypotheses 1 and 2). The differences in effect between linear and cubic interpolation alone are negligible and should be considered moot (hypothesis 3). In comparing these effects against a conventional low-pass filtering operation, additional context is obtained for the smallness of the effect on these features via interpolation (hypothesis 4). From this, we concluded as a primary finding that interpolation by itself is a relatively less effective operation on pressure array features, especially in comparison with filtering, and that there is a consistent effect of greater distortion of parameters following linear interpolation versus cubic interpolation, though this effect is weak and may well be small enough to overlook.

\section{Secondary Findings}

We also found that filtering interpolated data led to a significantly greater effect on the features extracted from data that were filtered (but not interpolated) than did the interpolation of already-filtered data (Table 3). On this basis, we concluded that it is very likely preferable to filter prior to interpolation. Given that there is a modest (but statistically significant) decrease in feature alteration via cubic interpolation (compared with linear interpolation) when implemented prior to filtering, we suggest that it may be preferred to select a cubic interpolation but advise that this is a tentative suggestion at best and that there is likely no substantive difference between the two approaches in this particular respect.

The perturbations to pressure map features following high-degree interpolations $(2 \times$ and $3 \times$ ) are incremental but small. In the worst-case scenario (mean pressure in the jump from linear $1 \times$ to linear $3 \times$ ), there is a 60 percent relative increase in effect size ( $9 \%$ decrease from raw in $1 \times$ vs $15 \%$ decrease from raw in $3 \times$ ). However, several parameters remain negligibly altered, remaining at 1 to 2 percent alteration at $3 \times$ interpolation (Table 4 ).

\section{Study Design}

This study was designed with an interest in maximum generalizability and interpretability by studying two common interpolation methods (linear vs cubic spline interpolation) for their effect on features extracted from the pressure map. Attention was paid to the effect of order of operations with regard to filtering and the effect of increasing interpolation degree $(1 \times$ to $2 \times$ and $3 \times)$; these are the design approaches that every investigator must consider when interpolating. Common approaches to study design were purposely selected and a ubiquitous filter design (Gaussian low-pass filter with $20 \%$ neighborhood block size) was used in order to maximize connection of this study to the broadest representation of existing literatures and relevance to those planning studies in the future. Moreover, data were analyzed from a cohort of patients without preexisting conditions that might unnaturally alter their seating profile and with an inclusive approach to seat cushions and other supports incorporated into the sitting, so long as there was minimal risk of creating measurement artifact.

Three simplistic measures were included in this study: maximum, mean, and RMS of the gradient of pressure within a matrix array (pressure map). While these features do not necessarily have relevance to every study, they (or similar measures) have a broad representation in the literature and have the advantage of high interpretability. It is not feasible to test every possible outcome measure, but many outcome measures incorporate some combination or modest variation of these measures, e.g., ratio of peak-to-average pressure [34], to say nothing of these features analyzed in aggregate over time [9,35]. Whereas there is no universally accepted method for the analysis of interface pressure data [36], we decided that incorporating easily understood features that would extend the interpretability and usefulness of this study.

\section{Study Limitations}

Our interpretations of the results born out of this study are heavily influenced by the choices of baseline comparators (raw data in most cases or filtered data in the case of order of operations). In this way, perhaps the most important limitation of this study is in the base assumption that "raw" is best and that processed data "should look like the raw." We acknowledge that in many instances this will be true, but not always. Interpolation is used both for prediction of unknown data values and for adapting sparse data for enhanced visualization. However, where visual clarity is prioritized over prediction accuracy, it seems probable that high-precision estimates of the effect of interpolation may not be useful to begin with, but that in the vast majority of instances where quantitative measure is sought, this study is relevant. Nevertheless, raw data are notoriously noisy (hence the 
near ubiquitous incorporation of low-pass filtering in the processing routine for these data). It raises the question: Does the raw data provide a useful benchmark for measuring the effect of interpolation? We believe the answer is "yes."

In the many studies where interpolation techniques are tested, the most common paradigm is cross-validation, i.e., temporary removal of a known data point from a complete data set and comparison of the predicted (interpolated) value to this ground truth; we refer the reader to two reviews for extended discussion on cross-validation in interpolation [37-38]. Based on this evidence, we believe that this study design is in good keeping with the conventions of the study of signal processing, both in biomedical application and beyond. Nevertheless, there is no community consensus on this matter: objective study of pressure array data is a still-growing field, and there are no other benchmark studies on which to base the design or to compare our findings. It is impossible to avoid having to make some assumption here, and this seemed the only reasonable design. We urge that the conclusions be understood in this context.

One particularly noteworthy technical limitation is that this study looks only at interpolations to "integer order," i.e., interpolations to $2 n-1$ data points. Other interpolation orders will most certainly change the outcome of the parameters. We believe that this is most likely to affect maximum pressure, according to whether the raw data are even or odd in number (e.g., 16 vs 15 sensors) and whether these data are interpolated to odd or even order (e.g., 16 sensors to 31 vs 32 data points). We believe that parameters not based on point-estimates, e.g., average pressure or gradient measures, will be much less sensitive to this variable.

\section{Implications}

This is the first study we know of to measure the effect of various data processing schemes on scalar measures derived from pressure map data. In this way, we propose that this work might well be interpreted as a benchmark study in pressure array signal processing and its outcomes as a first avenue toward a consensus "best practice." Naturally, any such approach would require both (1) general agreement of the assumption that fidelity to the raw signal is a meaningful target and (2) replication of these methods by other researchers and on other data sets. Nevertheless, this study establishes a simplistic framework on a generalizable data set by which a stan- dard approach might be undertaken. Additionally, this work opens (and provides a first answer) to the question of how many sensors are needed.

Furthermore, we note that this study is yet another reminder that every step of the data acquisition process and signal processing chain will change the nature of the data. Sometimes these effects are intended, and other times they are not. Sometimes the effect is to improve the signal quality, and other times the signal is corrupted. We advise that the choice of whether and how to interpolate is ultimately a decision that must be made during the inquiry process and that no benchmarking study can dictate how to approach this activity. However, it is our intention to inform the process of design for future studies and lend additional context for results that have already been published.

\section{Broader Effects}

While the expressed intention in this study is to better understand the role of interpolation in pressure array data in application to the seating interface, we note that the principles of this work have potentially far-ranging effect: force-sensitive resistors are used in a variety of measurement activities. Some of the biomedical applications include measurement of grip strength [39], monitoring prosthetic socket interface pressures [40], detection of gait parameters both via forces exerted at the bottom of the foot [41-42], and detection of the topography of the upper leg as the skeletal muscles contract [43]. It has been known for $15 \mathrm{yr}$ that detection of neuromuscular volition in prosthetic application is sufficiently robust to allow multiple degrees of freedom in control of a prosthetic hand [44-45]. Whereas each of these activities was developed using multiple pressure sensors in an array for which interpolation might be applied, we encourage investigators from outside the discipline of seat interface pressure measurement to incorporate this work into their own data processing and reproduce our study and report their own findings. We acknowledge that in spite of the growing use of pressure array data, there remain significant reservations in the scientific community about the use of quantitative data from pressure array systems.

\section{CONCLUSIONS}

Three aspects of pressure array data processing were tested, pursuant to a first-ever view into the sensitivity of 
pressure map features to interpolation and filtering in low-resolution data collected at the seating interface. From the base assumption that the interpolation step should have minimal effect on these features, we concluded that pressure map data that are to be interpolated (1) should be filtered prior to interpolating; (2) can be interpolated via either a linear or cubic interpolation (with a possible slight preference to cubic interpolation); and (3) should have a small, but not substantial, effect on feature extraction due to increase in interpolation degree through an interpolation to an $8 \times$ sampling density. We invite other investigators to perform similar benchmark analysis on their own data, in the interest of establishing a community consensus of best practices in pressure array data processing.

\section{ACKNOWLEDGMENTS}

\section{Author Contributions:}

Study concept and design: B. Crane, M. Wininger.

Acquisition of data: B. Crane.

Analysis and interpretation of data: M. Wininger.

Statistical analysis: M. Wininger.

Drafting of manuscript: M. Wininger.

Critical revision of manuscript for important intellectual content:

M. Wininger, B. Crane.

Financial Disclosures: The authors have declared that no competing interests exist.

Funding/Support: This material was unfunded at the time of manuscript preparation.

Institutional Review: All human subject procedures were approved by the University of Hartford Human Subjects Committee and the Genesis Health Care System Institutional Review Board prior to any recruitment or data collection activities. All participants completed the informed consent process and signed informed consent documents prior to any data collection.

Participant Follow-Up: The authors do not plan to inform participants of the publication of this study.

\section{REFERENCES}

1. Ferguson-Pell M, Cardi MD. Prototype development and comparative evaluation of wheelchair pressure mapping system. Assist Technol. 1993;5(2):78-91.

[PMID:10146318]

http://dx.doi.org/10.1080/10400435.1993.10132213

2. Keast DH, Parslow N, Houghton PE, Norton L, Fraser C. Best practice recommendations for the prevention and treatment of pressure ulcers: Update 2006. Adv Skin Wound Care. 2007;20(8):447-60, quiz 461-62.

\section{[PMID:17762312]}

http://dx.doi.org/10.1097/01.ASW.0000284922.69932.c5

3. Reenalda J, Van Geffen P, Nederhand M, Jannink M, IJzerman M, Rietman $\mathrm{H}$. Analysis of healthy sitting behavior: Interface pressure distribution and subcutaneous tissue oxygenation. J Rehabil Res Dev. 2009;46(5):577-86.

[PMID:19882492]

http://dx.doi.org/10.1682/JRRD.2008.12.0164

4. Giesbrecht EM, Ethans KD, Staley D. Measuring the effect of incremental angles of wheelchair tilt on interface pressure among individuals with spinal cord injury. Spinal Cord. 2011;49(7):827-31. [PMID:21242997] http://dx.doi.org/10.1038/sc.2010.194

5. Makhsous M, Rowles DM, Rymer WZ, Bankard J, Nam EK, Chen D, Lin F. Periodically relieving ischial sitting load to decrease the risk of pressure ulcers. Arch Phys Med Rehabil. 2007;88(7):862-70. [PMID:17601466] http://dx.doi.org/10.1016/j.apmr.2007.03.017

6. Stinson MD, Porter-Armstrong A, Eakin P. Seat-interface pressure: A pilot study of the relationship to gender, body mass index, and seating position. Arch Phys Med Rehabil. 2003;84(3):405-9. [PMID:12638109] http://dx.doi.org/10.1053/apmr.2003.50011

7. Grondin DE, Triano JJ, Tran S, Soave D. The effect of a lumbar support pillow on lumbar posture and comfort during a prolonged seated task. Chiropr Man Therap. 2013; 21(1):21. [PMID:23826832] http://dx.doi.org/10.1186/2045-709X-21-21

8. Triolo RJ, Bailey SN, Lombardo LM, Miller ME, Foglyano $\mathrm{K}$, Audu ML. Effects of intramuscular trunk stimulation on manual wheelchair propulsion mechanics in 6 subjects with spinal cord injury. Arch Phys Med Rehabil. 2013;94(10): 1997-2005. [PMID:23628377] http://dx.doi.org/10.1016/j.apmr.2013.04.010

9. Vandewalle P, Kovacevic J, Vetterli M. Reproducible research in signal processing. IEEE Signal Process Mag. 2009;26(3):37-47. http://dx.doi.org/10.1109/MSP.2009.932122

10. Weiner N. Extrapolation, interpolation, and smoothing of stationary time series, with engineering applications. Cambridge (MA): Technology Press of Massachusetts Institute of Technology; 1949.

11. Treaster D, Marras WS. Measurement of seat pressure distributions. Hum Factors. 1987;29(5):563-75.

12. Robinson AP, Hamann JD. Forest analytics with R: An introduction. New York (NY): Springer; 2011. p. 117-51.

13. Brienza DM, Karg PE, Geyer MJ, Kelsey S, Trefler E. The relationship between pressure ulcer incidence and buttockseat cushion interface pressure in at-risk elderly wheelchair users. Arch Phys Med Rehabil. 2001;82(4):529-33.

[PMID:11295017]

http://dx.doi.org/10.1053/apmr.2001.21854 
14. Sprigle S, Dunlop W, Press L. Reliability of bench tests of interface pressure. Assist Technol. 2003;15(1):49-57. [PMID:14760981] http://dx.doi.org/10.1080/10400435.2003.10131889

15. Sprigle S, Sonenblum S. Assessing evidence supporting redistribution of pressure for pressure ulcer prevention: A review. J Rehabil Res Dev. 2011;48(3):203-13. [PMID:21480095] http://dx.doi.org/10.1682/JRRD.2010.05.0102

16. Tam EW, Mak AF, Lam WN, Evans JH, Chow YY. Pelvic movement and interface pressure distribution during manual wheelchair propulsion. Arch Phys Med Rehabil. 2003;84(10):1466-72. [PMID:14586913] http://dx.doi.org/10.1016/S0003-9993(03)00269-7

17. Andreoni G, Pedotti A, Ferrarin M. Pressure distribution on wheelchair cushions in static sitting and during manual propulsion. J Mech Med Biol. 2001;1(1):33-44. http://dx.doi.org/10.1142/S0219519401000076

18. Meffre R, Gehin C, Dittmar A. MAPI: Active interface pressure sensor integrated into a seat. Proceedings of the 29th Annual International Conference of the IEEE Engineering in Medicine and Biology Society; 2007 Aug 2226; Lyon, France.

19. Moes NC. Variation in sitting pressure distribution and location of the points of maximum pressure with rotation of the pelvis, gender and body characteristics. Ergonomics. 2007;50(4):536-61. [PMID:17575714] http://dx.doi.org/10.1080/00140130601138585

20. Ferrarin M, Andreoni G, Pedotti A. Comparative biomechanical evaluation of different wheelchair seat cushions. J Rehabil Res Dev. 2000;37(3):315-24. [PMID:10917263]

21. Marenzi E, Bertolotti GM, Cristiani A, De Donno F, Leporati $\mathrm{F}$, Danese $\mathrm{G}$, Bejor $\mathrm{M}$. Identification and evaluation of parameters for the prevention of pressure ulcers in hospitalized patients. Proceedings of the 2013 IEEE EUROCON; 2013 Jul 1-4; Zagreb, Croatia.

22. Solis LR, Liggins A, Uwiera RR, Poppe N, Pehowich E, Seres P, Thompson RB, Mushahwar VK. Distribution of internal pressure around bony prominences: Implications to deep tissue injury and effectiveness of intermittent electrical stimulation. Ann Biomed Eng. 2012;40(8):1740-59.

[PMID:22354272] http://dx.doi.org/10.1007/s10439-012-0529-0

23. Helsingius M, Haavisto P. Interpolating the HDMAC signal to produce high quality still images. Proceedings of the 1993 IEEE Winter Workshop on Nonlinear Digital Signal Processing; 1993 Jan 17-20; Tampere, Finland.

24. Shao Y, Wang ZZ, Zhang YM. Monitoring of liquid droplets in laser-enhanced GMAW. Int J Adv Manuf Technol. 2011;57(1-4):203-14. http://dx.doi.org/10.1007/s00170-011-3266-X
25. Wang C, Zhang L, He Y, Tan YP. Frame rate up-conversion using trilateral filtering. IEEE Trans Circuits Syst Video Technol. 2010;20(6):886-93.

http://dx.doi.org/10.1109/TCSVT.2010.2046057

26. Griffiths HD, Baker CJ. Fundamentals of tomography and radar. In: Byrnes J, editor. Advances in sensing with security applications. Dordrecht (the Netherlands): Springer; 2006. p. 171-87.

27. Yang SC, Kalnay E, Hunt BE, Bowler N. Weight interpolation for efficient data assimilation with the Local Ensemble Transform Kalman Filter. Q J R Meteorol Soc. 2009; 135(638):251-62. http://dx.doi.org/10.1002/qj.353

28. Yang YH, Wang PH, Chen PN, Wu CL. An edge-preserving interpolation in CCD color filter arrays. Proceedings of the 2010 IEEE International Conference on Imaging Systems and Techniques; 2010 Jul 1-2; Thessaloniki, Greece.

29. Crawford SA, Stinson MD, Walsh DM, Porter-Armstrong AP. Impact of sitting time on seat-interface pressure and on pressure mapping with multiple sclerosis patients. Arch Phys Med Rehabil. 2005;86(6):1221-25.

[PMID:15954063]

http://dx.doi.org/10.1016/j.apmr.2004.08.010

30. Aissaoui R, Lacoste M, Dansereau J. Analysis of sliding and pressure distribution during a repositioning of persons in a simulator chair. IEEE Trans Neural Syst Rehabil Eng. 2001;9(2):215-24. [PMID:11474974] http://dx.doi.org/10.1109/7333.928581

31. Hall RS, Desmoulin GT, Milner TE. A technique for conditioning and calibrating force-sensing resistors for repeatable and reliable measurement of compressive force. J Biomech. 2008;41(16):3492-95. [PMID:19019374] http://dx.doi.org/10.1016/j.jbiomech.2008.09.031

32. Lebosse C, Renaud P, Bayle B, de Mathelin M. Modeling and evaluation of low-cost force sensor. IEEE Trans Robot. 2011;27(4):815-22. http://dx.doi.org/10.1109/TRO.2011.2119850

33. Vecchi F, Freschi C, Micera S, Sabatini AM, Dario P, Sacchetti R. Experimental evaluation of two commercial force sensors for applications in biomechanics and motor control. Proceedings of the 5th Annual Conference of the International Functional Electrical Stimulation Society; 2000; Aalborg, Denmark.

34. Rithalia S. Assessment of patient support surfaces: Principle, practice and limitations. J Med Eng Technol. 2005; 29(4):163-69. [PMID:16012067] http://dx.doi.org/10.1080/03091900410001731191

35. Mushahwar VK, Solis-Aguilar LR, inventors; The Governors of the University of Alberta, assignee. Mitigation of pressure ulcers using electrical stimulation. United States patent US20110112604 A1. 2011 May 12.

36. Aissaoui R, Kauffmann C, Dansereau J, de Guise JA. Analysis of pressure distribution at the body-seat interface in able-bodied 
and paraplegic subjects using a deformable active contour algorithm. Med Eng Phys. 2001;23(6):359-67.

[PMID:11551812]

http://dx.doi.org/10.1016/S1350-4533(01)00052-2

37. Escandar GM, Damiani PC, Goicoechea HC, Olivieri AC. A review of multivariate calibration methods applied to biomedical analysis. Microchem J. 2006;82(1):29-42. http://dx.doi.org/10.1016/j.microc.2005.07.001

38. Hanzer F. Spatial interpolation of scattered geoscientific data [Internet]. 2012 Feb 3. Available from: http://www.unigraz.at/ haasegu/Lectures/GPU CUDA/WS11/ hanzer_presentation2.pdf

39. Kim NH, Wininger M, Craelius W. Training grip control with a Fitts' paradigm: A pilot study in chronic stroke. J Hand Ther. 2010;23(1):63-72. [PMID:20142007] http://dx.doi.org/10.1016/j.jht.2009.10.004

40. Buis AW, Convery P. Calibration problems encountered while monitoring stump/socket interface pressures with force sensing resistors: Techniques adopted to minimise inaccuracies. Prosthet Orthot Int. 1997;21(3):179-82. [PMID:9453089]

41. Pappas IP, Popovic MR, Keller T, Dietz V, Morari M. A reliable gait phase detection system. IEEE Trans Neural Syst Rehabil Eng. 2001;9(2):113-25. [PMID:11474964]

42. Zehr EP, Stein RB, Komiyama T, Kenwell Z. Linearization of force sensing resistors (FSR's) for force measurement during gait. Proceedings of the IEEE 17th Annual Conference on Engineering in Medicine and Biology Society; 1995 Sep 20-23; Montreal, Canada.

43. Yungher DA, Wininger MT, Barr JB, Craelius W, Threlkeld AJ. Surface muscle pressure as a measure of active and passive behavior of muscles during gait. Med Eng Phys. 2011;33(4):464-71. [PMID:21176884]

http://dx.doi.org/10.1016/j.medengphy.2010.11.012

44. Phillips SL, Craelius W. Residual kinetic imaging: A versatile interface for prosthetic control. Robotica. 2005;23(3): 277-82. http://dx.doi.org/10.1017/S0263574704001298

45. Wininger M, Kim N-H, Craelius W. Pressure signature of forearm as predictor of grip force. J Rehabil Res Dev. 2008;45(6):883-92. [PMID:19009474] http://dx.doi.org/10.1682/JRRD.2007.11.0187

Submitted for publication April 10, 2014. Accepted in revised form July 31, 2014.

This article and any supplementary material should be cited as follows:

Wininger M, Crane B. Effect of interpolation on parameters extracted from seating interface pressure arrays. J Rehabil Res Dev. 2014;51(9):1365-76.

http://dx.doi.org/10.1682/JRRD.2014.04.0101

ResearcherID/ORCID: Michael Wininger, PhD: 00000002-7871-3292; Barbara Crane, PhD, PT: 0000-00017826-9243

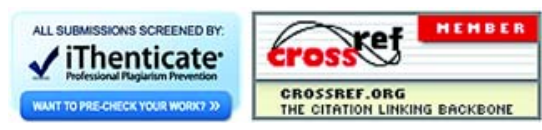


